Article

\title{
Optimizing the Preparation of Semi-Crystalline Paraffin/Poly(Urea-Formaldehyde) Microcapsules for Thermal Energy Storage
}

\author{
Kai Zhang ${ }^{1}$, Qian Zhou ${ }^{1, *}$ and Hai-Mu Ye ${ }^{2, * \mathbb{D}}$ \\ 1 Department of Applied Chemistry, China University of Petroleum, Beijing 102249, China; \\ zhangkai037@163.com \\ 2 Department of Materials Science \& Engineering, China University of Petroleum, Beijing 102249, China \\ * Correspondence: zqlqt@163.com (Q.Z.); yehaimu@cup.edu.cn (H.-M.Y.)
}

Received: 5 January 2019; Accepted: 8 February 2019; Published: 12 February 2019

check for updates

\begin{abstract}
Paraffin, the most common phase change material, has been widely utilized as the core component in thermal energy storage in the form of microcapsules. In this study, semi-crystalline paraffin is capsulated into a poly(urea-formaldehyde) (PUF) shell by a two-step polymerization process. To obtain the microcapsule with good morphology and high latent heat, sodium chloride and crosslinker (a mixture of ammonium chloride and resorcinol with a weight ratio of 1:1) are incorporated and their addition amounts were optimized through differential scanning calorimetry (DSC) and SEM. The optimized microcapsules were obtained by adding $4 \mathrm{wt} \%$ sodium chloride, and $0.25 \mathrm{wt} \%$ crosslinker exhibits a diameter of several microns and a melting enthalpy of $110 \mathrm{~J} / \mathrm{g}$. This detailed study shows that sodium chloride strongly affects the morphology of paraffin emulsion by enlarging droplets, widening the size distribution, and enhancing the stability, which should be attributed to the enhancement of electric double layer strength. In addition, sodium chloride can weaken the Zeta potential of prepolymer and provides more opportunity for prepolymer to deposit on the surface of emulsion droplets. The two components in crosslinker play different roles in the polymerization process. Ammonium chloride reacts with prepolymers and reduces the $\mathrm{pH}$ of system, which can accelerate the curing process, while resorcinol probably participates in polymerization as a comonomer.
\end{abstract}

Keywords: Paraffin; Poly(urea-formaldehyde); Phase change microcapsules; Sodium chloride; Crosslinker

\section{Introduction}

Energy requirements are increasing rapidly due to fast population growth. The burning of fossil fuels with emissions of harmful gases has brought severe damage to that environment and has caused global warming [1]. To solve the accelerating pollution from consumption of traditional fossil fuels, scientists are focusing on improving both the efficiency of utilization and researching renewable resources [2]. Solar, the source of most energy on earth, has almost infinite reserves and can be a promising alternative. However, a large part of solar energy is converted to other forms and wasted during transition. Moreover, solar is not effectively controllable and their exits a mismatch between availability and utilization [3]. Therefore, appropriate thermal energy storage (TES) systems are needed to conserve the waste energy. The use of TES in a solar system can promote overall efficiency, reduce investment, decrease running costs, and have a positive impact on the environment [4].

Excess solar can be stored in the daytime by TES devices, and then used at night. Typically, the forms of TES include: Sensible heat, latent heat, chemical reaction heat storage, and latent heat storage (LHS). The LHS system is considered to be the most promising method with providing huge potential 
for the storage of solar energy [5]. Compared with sensible heat storage (SHS), the process of LHS saving and releasing thermal energy is based on the heat of solidification and fusion when the medium undergoes phase transformation [6]. The medium is called phase change materials (PCMs) and the heat during phase change is latent heat. The PCMs usually have constant melting and crystallization temperatures and offer large energy storage density within a limited temperature change, which has made PCMs applied in various fields, such as building, refrigeration, textile, automobile, space, and food industry [7-14].

Paraffin, with the chemical formula of $\mathrm{C}_{n} \mathrm{H}_{2 n+2}$, possesses the traits of high latent heat, low supercooling degree, and is non-corrosive. Moreover, the melting point and latent heat increase with the increasing value of carbon atom number, which make paraffin well known as an organic PCM. In most instances, paraffin used for solar TES is required to have a melting point between 50 and $60{ }^{\circ} \mathrm{C}$ [15]. Considering economic benefits and safety, thermal conductivity, density, degradation, volume change during phase transformation, toxicity, and cost are all important performance factors [16]. Microcrystalline wax meets most of the above performance factors, and the volume change that occurs as the working temperature increases can be completely controlled by microencapsulation techniques, which also prevents leakage and enlarges the heat transfer area [17]. Belessiotis et al. used a sol-gel method to synthesize paraffin $/ \mathrm{SiO}_{2}$ microcapsules, which possessed the highest capsulation ratio of $78.1 \%$ and a latent heat of $156.0 \mathrm{~J} / \mathrm{g}$ [18]. Li et al. prepared paraffin $/ \mathrm{SiO}_{2}$ microcapsules by in-situ polymerization with a melting temperature of $56.5^{\circ} \mathrm{C}$ and enthalpy of $45.5 \mathrm{~J} / \mathrm{g}$ [19]. Wei et al. investigated novel paraffin/polyurethane microcapsules prepared by interfacial polymerization with isophorone diisocyanate and ethylene diamine and demonstrated that the latent heat could reach $95.5 \mathrm{~J} / \mathrm{g}$ when the core/shell ratio was 75/25 [20]. Moreover, physical methods, including spray drying and solvent evaporation, also can be used in microencapsulation [21,22].

The shell is another important component of microcapsules. Urea-formaldehyde stands out from numerous shell materials due to it being a cheap raw material, convenient in regards to preperation preparation, having low toxicity, and good thermal conductivity [23]. Gaitzsch et al. summed up the methods of fabricating and designing confined microenvironments and the relevant applications after the first publications of Discher and Eisenberg on polymer vesicles [24]. Cuomo et al. focused on the preparation and designation of compartmentalized spaces as nanoreactor which could be used in biological and technical aspects [25]. Preparation and influencing factors of paraffin/poly(urea-formaldehyde) (PUF) microcapsules by in-situ polymerization have been investigated by many researchers [26]. Sánchez-Silva et al. have shown that almost no paraffin was capsulated in the shell prepared through a one-step method, while paraffin/PUF microcapsules was successfully fabricated by a two-step method with the poly(ethylene-alt-maleic anhdride) (PEMA) as emulsifier [27]. Longer prepolymerization and polymerization time can improve the morphology and capsulated paraffin content. Jin et al. prepared paraffin/PUF capsules with styrene-maleic anhydride alternating the copolymer as emulsifier and fabrication parameters, such as the amount of emulsifier and urea-formaldehyde (UF) precursor to optimize the performance of the product [28]. Xin et al. investigated the effect of curing temperature on the performance of paraffin/PUF capsules and presented the optimum curing temperature as $65^{\circ} \mathrm{C}$, leading to microcapsule enthalpy of $74.2 \mathrm{~J} / \mathrm{g} \mathrm{[29]}$. Yan et al. found that the addition of sodium chloride could increase the crystallization enthalpy from 65.0 to $79.8 \mathrm{~J} / \mathrm{g}$ and the morphologies of capsules could be significantly improved by adding the dispersing agent 2-Naphthalenesulfonic acid in the latter part of polymerization [30]. Jian et al. fabricated paraffin/PUF microcapsules with melting enthalpy of $117.6 \mathrm{~J} / \mathrm{g}$ and found that sodium chloride could improve the microcapsule morphology [31]. Fang et al. proved that mass ratio of core material increased from $30.4 \%$ to $61.8 \%$ with the resorcinol concentration increasing from $0.25 \%$ to $5 \%$. They also found that the morphologies became worse if the amount of resorcinol kept increasing [32]. Fan et al. found that the addition of ammonium could cause the $\mathrm{pH}$ of system to decrease, because of the reaction between ammonium chloride and formaldehyde. In addition, the reaction between 
ammonium chloride and urea-formaldehyde can generate active substances on the surface, which drives the formation of PUF nanoparticles to enrich on the surface of microcapsules [33].

In previous reports, many factors in the fabrication of microcapsules have been investigated. Sodium chloride and crosslinker have also been commonly used to improve the properties and performance of microcapsules. However, the influence of the amount of the two types of agents and how their working mechanisms are impacted has received little research attention and requires further study. This study aims to investigate the synthesis route of microcrystalline paraffin/PUF microcapsules and to find out the optimal technological parameters. In particular, the effects of sodium chloride on morphology and encapsulation efficiency are studied in detail.

\section{Experimental}

\subsection{Materials}

Paraffin with a melting point of about $58^{\circ} \mathrm{C}$ was provided by Tianjin Fuchen Chemical Reagents Factory. Formaldehyde solution (37 $\mathrm{wt} \%$ aqueous solution) was provided by Tianjin Fengchuan Chemical Reagent Company. Urea (AR grade), triethanolamine (AR grade), citric acid monohydrate (GR grade), OP-10, sodium chloride (AR grade), 1-pentanol (AR grade), resorcinol (AR grade), ammonium chloride (PT grade), and petroleum ether (AR grade) were all purchased from Shanghai Aladdin Company. All chemicals were used without further purification.

\subsection{Methods}

The reactions that occurred during the preparation of the microcapsules are shown in Scheme 1. Firstly, approximate amounts of urea and formaldehyde and $10 \mathrm{~mL}$ deionized water were added in a three-mouth flask with a stirring rate of $200 \mathrm{rpm}$. After the complete dissolution of monomers, $10 \mathrm{wt} \%$ triethanolamine solution was used to adjust the $\mathrm{pH}$ to 9 . The reaction was kept at $70{ }^{\circ} \mathrm{C}$ for $60 \mathrm{~min}$, and prepolymer solution was obtained. In this stage, formula (a) presented in the alkaline forms monomethylol (A) and dimethylol urea (B) and the main products were in accordance with the molar ratio of formaldehyde and urea. Secondly, paraffin, OP-10 and $200 \mathrm{~mL}$ deionized water were emulsified in another three-necked flask by mechanical stirring with a stirring rate of $1000 \mathrm{rpm}$ for $40 \mathrm{~min}$. With adding drops of 1-pentanol to eliminate bubbles [34], the paraffin emulsion was prepared. Following this, the stirring rate was reduced to $500 \mathrm{rpm}$ and sodium chloride solution was added within $5 \mathrm{~min}$ followed by the slow addition of prepolymer solution in $15 \mathrm{~min}$. $10 \mathrm{wt} \%$ citric acid was used to adjust the $\mathrm{pH}$ of solution to 3 to 4 and the reaction began at $65^{\circ} \mathrm{C}$. Formula (b), (c), and (d) generated the crosslinked structure (C). If the formaldehyde still existed or the $\mathrm{pH}$ was adjusted too quickly, reactions (e), (f), and (g) might happen. Finally, crosslinker was added $2 \mathrm{~h}$ later to help curing and the wanted products were obtained after another $2 \mathrm{~h}$ and were washed twice with petroleum ether and water respectively. Following this, they were filtered and dried in a vacuum oven for $24 \mathrm{~h}$.

\subsection{Characterization}

The latent heat and melting points of paraffin and microcapsules were characterized by differential scanning calorimetry (DSC) and the measurement was performed on a NETZSCH 204F1 instrument under nitrogen atmosphere equipped with an intercooler as the cooling system. Heating and cooling were set at a rate of $10{ }^{\circ} \mathrm{C} / \mathrm{min}$ in temperature range of 0 to $100^{\circ} \mathrm{C}$. The encapsulation efficiency (E) of paraffin was calculated by the following equation:

$$
E=\frac{\Delta H_{m, m}}{\Delta H_{m, p}} \times 100 \%
$$

where $\Delta H_{\mathrm{m}, \mathrm{m}}$ and $\Delta H_{\mathrm{m}, \mathrm{p}}$ represent the latent heat of the microcapsules and paraffin, respectively. 
The chemical structure of PUF, paraffin, and the microcapsules were studied by using a Bruker Tension II Fourier transform infrared (FTIR) spectrometer with a time resolution of $4 \mathrm{~cm}^{-1}$. The morphology and particle size of the capsules were characterized using a Hitachi SU8010 scanning electron microscopy (SEM) with an acceleration voltage of $5 \mathrm{kV}$. The dispersion state and stability of emulsified paraffin droplets was observed using an Anton Paar Letesizer-500 particle size analyzer (PSA).

In alkaline :

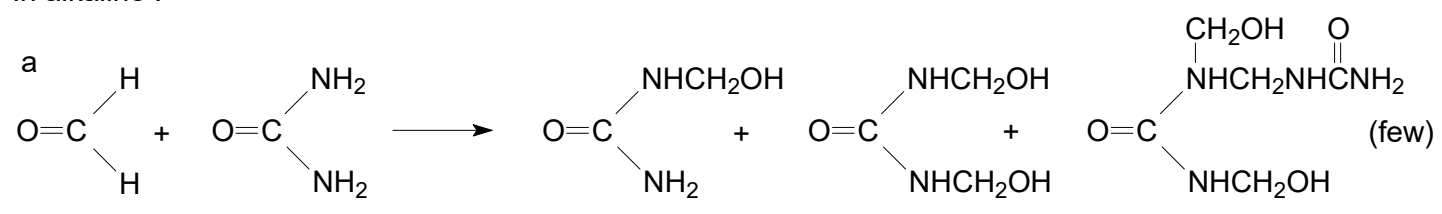

(A)

(B)

In acidity :

b<smiles>CN(CO)C(=O)N(C)CO</smiles><smiles>CNC(=O)N(C)C=O</smiles>

c<smiles>CN(C)C(=O)N(CO)[14CH2]N(C)C(=O)N(C)CO</smiles><smiles>CNCC(=O)NC</smiles>

d

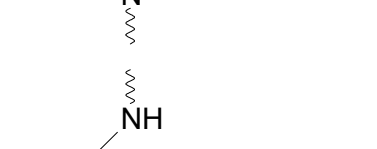<smiles>C1CCC1</smiles><smiles>CCCC</smiles>

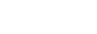




\section{Results and Discussion}

\subsection{Performance of Microcapsules without Sodium Chloride and Crosslinker}

Paraffin/PUF microcapsules without the addition of sodium and crosslinker were first prepared and characterized as the control. As shown in Figure 1, the microcapsules exhibited a melting point of $57.7^{\circ} \mathrm{C}$ and melting enthalpy of $42.8 \mathrm{~J} / \mathrm{g}$. By comparing to melting enthalpy of raw microcrystalline paraffin $(185.2 \mathrm{~J} / \mathrm{g})$, the encapsulation efficiency of paraffin was only $23.1 \%$. However, the microcapsules showed higher crystallization temperature than raw paraffin during cooling process, $54.6{ }^{\circ} \mathrm{C}$ vs. $50.8^{\circ} \mathrm{C}$, which benefited the fast release of latent heat. The improvement of crystallization behavior might originate from two aspects. On one hand, PUF possibly plays the role of nucleating agent for paraffin. On the other hand, the interface between PUF and paraffin with curve geometry could improve the primary nucleation of paraffin [35-37].

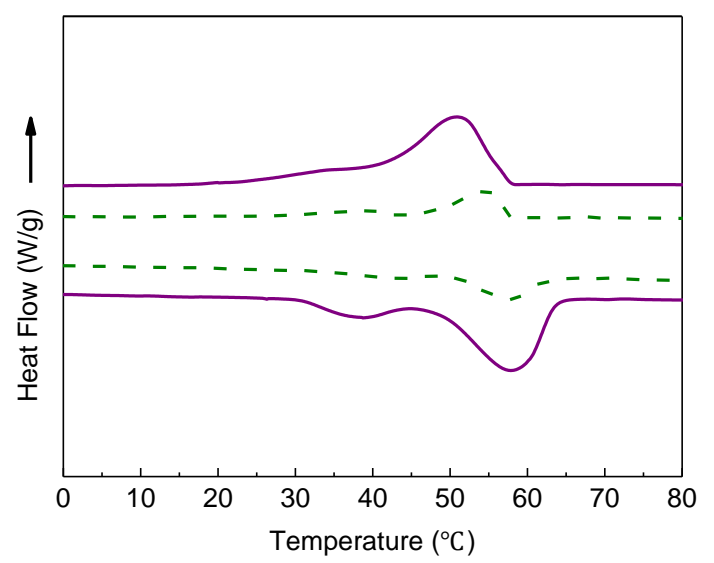

Figure 1. Differential scanning calorimetry (DSC) heating and cooling curves of paraffin (solid line) and microcapsules prepared without sodium chloride and crosslinker (dash line).

To directly visualize the successful encapsulation of paraffin by PUF, both the as-prepared product and raw paraffin were placed on a hot stage prefixed at temperature of $70{ }^{\circ} \mathrm{C}$. Figure 2 shows that the raw paraffin immediately melted as soon as it was put on the hot-stage. However, the appearance of the as-prepared microcapsules stayed unchanged, and no liquid leakage occurred even it has been heated for over $20 \mathrm{~min}$. Thus, the paraffin was well accommodated by the compact PUF shell.
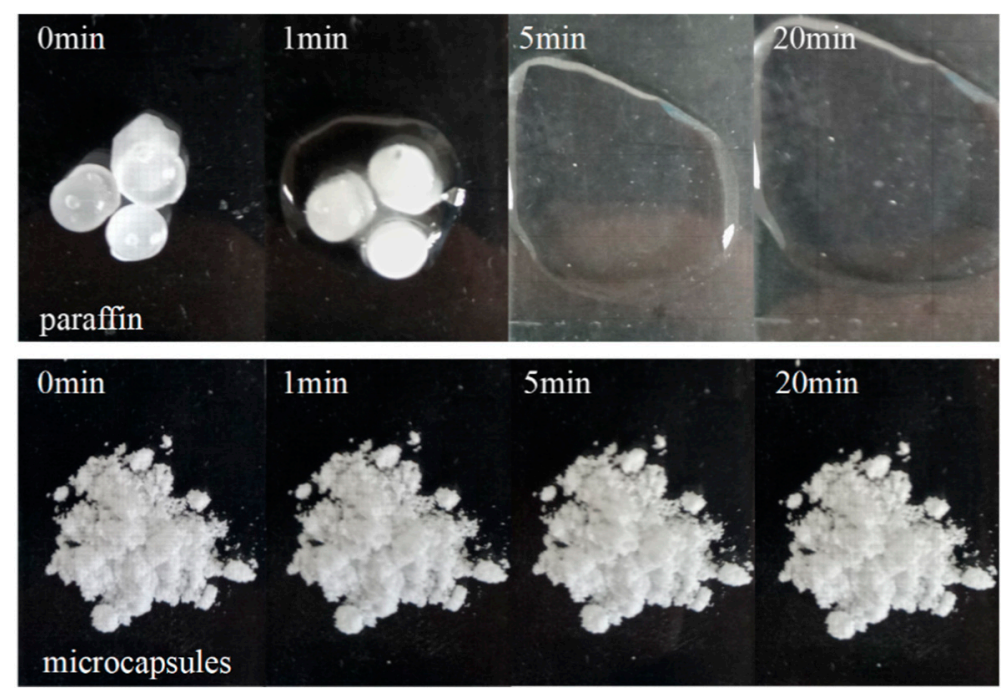

Figure 2. Photographs of the evolution of raw paraffin and paraffin/PUF microcapsules on hot-stage fixed at $70{ }^{\circ} \mathrm{C}$. 
The FTIR spectra of paraffin, PUF, and the microcapsules are shown in Figure 3. Spectra (a) and (b) show strong multiple absorption bands between 3000 and $2800 \mathrm{~cm}^{-1}$, which are associated with the aliphatic $\mathrm{C}-\mathrm{H}$ stretching vibrations, while those of PUF are relatively weak in spectra (c). The absorption band at $1464 \mathrm{~cm}^{-1}$ is assigned to the $\mathrm{C}-\mathrm{H}$ bending vibrations and the band at $722 \mathrm{~cm}^{-1}$ is attributed to the groups of $\mathrm{CH}_{2}$ inplane vibrations. The characteristic peaks mentioned above belong to the core material, paraffin. The broad band at $3350 \mathrm{~cm}^{-1}$ is assigned to $\mathrm{N}-\mathrm{H}$ stretching vibrations and it shifts because the hydrogen of amide participates in polymerization, which is unfavorable for the stretching vibration [38] The absorption bands at 1637 and $1543 \mathrm{~cm}^{-1}$, which can be observed in spectra (a) and (c), are attributed to the $\mathrm{C}=\mathrm{O}$ and $\mathrm{C}-\mathrm{N}$ stretching vibrations of amide, respectively. Bands between 1377 and $1024 \mathrm{~cm}^{-1}$, belonging to amide III are caused by the multiple reaction of active hydrogen of amide [39] These absorption bands represent the PUF shell materials. Through the above analysis, characteristic bands of paraffin and PUF can be found in spectra of microcapsules and almost no extra bands appear, which infers that no chemical reaction happens between the core and shell. The paraffin is well encapsulated by PUF shell physically.

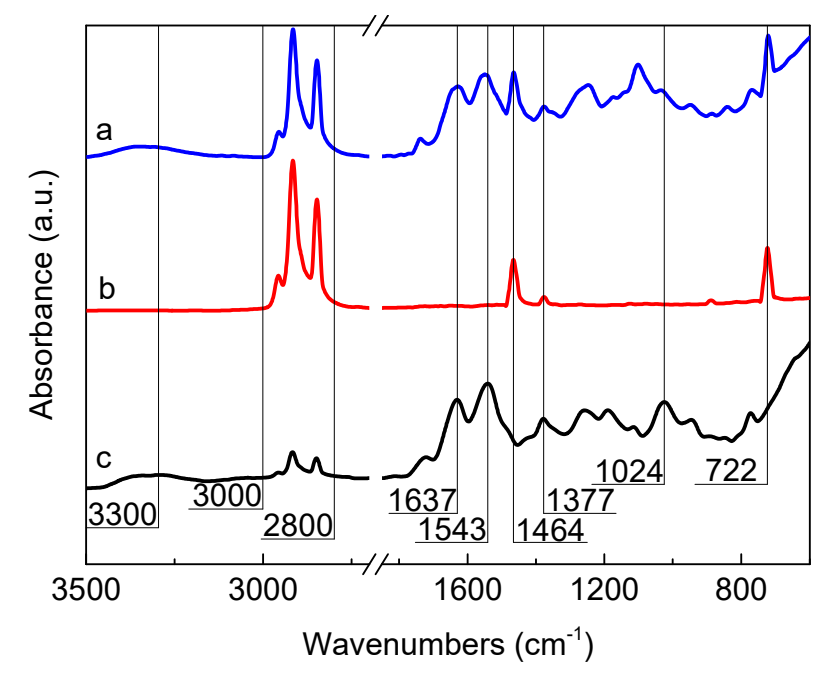

Figure 3. FTIR spectra of paraffin/PUF microcapsules (a), raw paraffin (b), and PUF (c).

\subsection{Optimizing Microcapsule Performance by Adding Sodium Chloride}

Although the above result has demonstrated that paraffin is encapsulated by PUF shell, the SEM image in Figure 4A highlights that the morphology of the microcapsules was not ideal. Lumps of microcapsules with size of several tens microns are observed and small particles stacked on the rough surface, which is similar to previous reported paraffin/PUF microcapsules without the assistant of sodium chloride and crosslinker [40]. The poor morphology corresponds to the low latent heat and encapsulation efficiency.

To improve the morphology of the microcapsule, sodium chloride is widely used [41]. The typical morphologies of paraffin/PUF microcapsules prepared with different addition amounts of sodium chloride are shown in Figure 4B-E. Once the sodium chloride was introduced, the morphology of the microcapsules became regular. When the addition of sodium chloride reached $1 \mathrm{wt} \%$, the microcapsules were still bonded to each other but the tendency of separation was clearer. Apparent spherical morphology of microcapsules emerged after increasing the sodium chloride amount to $2 \mathrm{wt} \%$, and the diameter ranged from 2 to $5 \mu \mathrm{m}$. With an increased sodium chloride content of $4 \mathrm{wt} \%$ and $8 \mathrm{wt} \%$, spherical and independent microcapsules with a smooth surface were found. Some small particles adhered on the surface of the microcapsules, and this was due to the self-polymerization of PUF [33]. If the addition of sodium chloride is further increased to $16 \mathrm{wt} \%$, demulsification occurred and the obvious boundary can be observed between the water and paraffin phase. 
Figure 5 depicts the DSC curves of paraffin/PUF microcapsules with different amounts of sodium chloride. The melting enthalpy of the microcapsules increased when sodium chloride was increased and reached a maximum value of $67.5 \mathrm{~J} / \mathrm{g}$ at $4 \mathrm{wt} \%$ sodium chloride content, the equivalent to a paraffin encapsulation efficiency of $36.4 \%$. Increased addition of sodium chloride led to a significant decline in melting enthalpy. For instance, the melting enthalpy of the microcapsules prepared with $8 \mathrm{wt} \%$ sodium chloride dropped to $44.6 \mathrm{~J} / \mathrm{g}$, which is even close to the microcapsules obtained without the addition of sodium chloride. Thus, the addition of sodium can help encapsulate paraffin, but an excess dose of sodium chloride will a show remarkably negative effect.

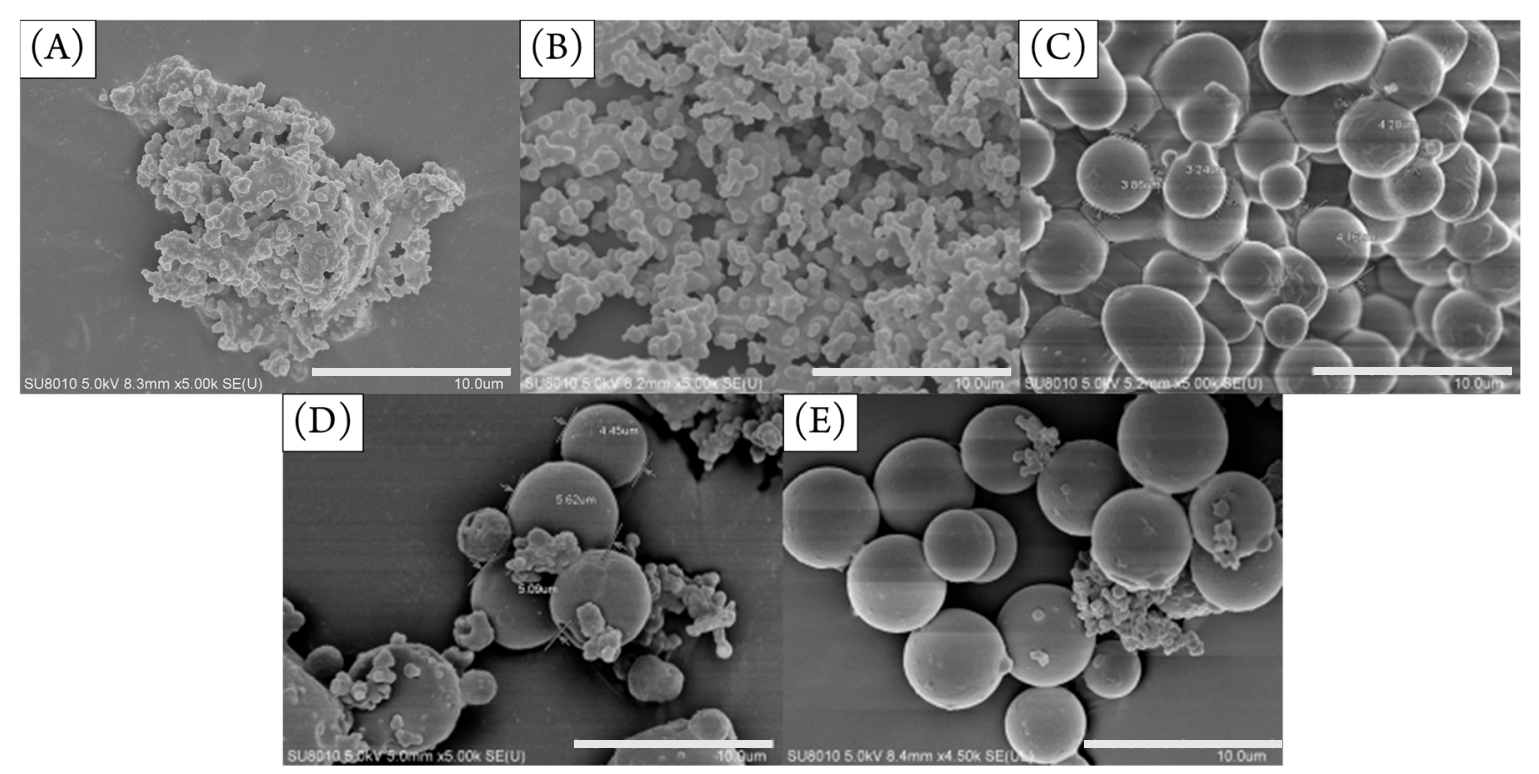

Figure 4. SEM images of microcapsules prepared with different addition amounts of sodium chloride. 0 (A), $1 \mathrm{wt} \%($ B), $2 \mathrm{wt} \%(\mathbf{C}), 4 \mathrm{wt} \%$ (D), and $8 \mathrm{wt} \%$ (E). The scale bars are all $10 \mu \mathrm{m}$.
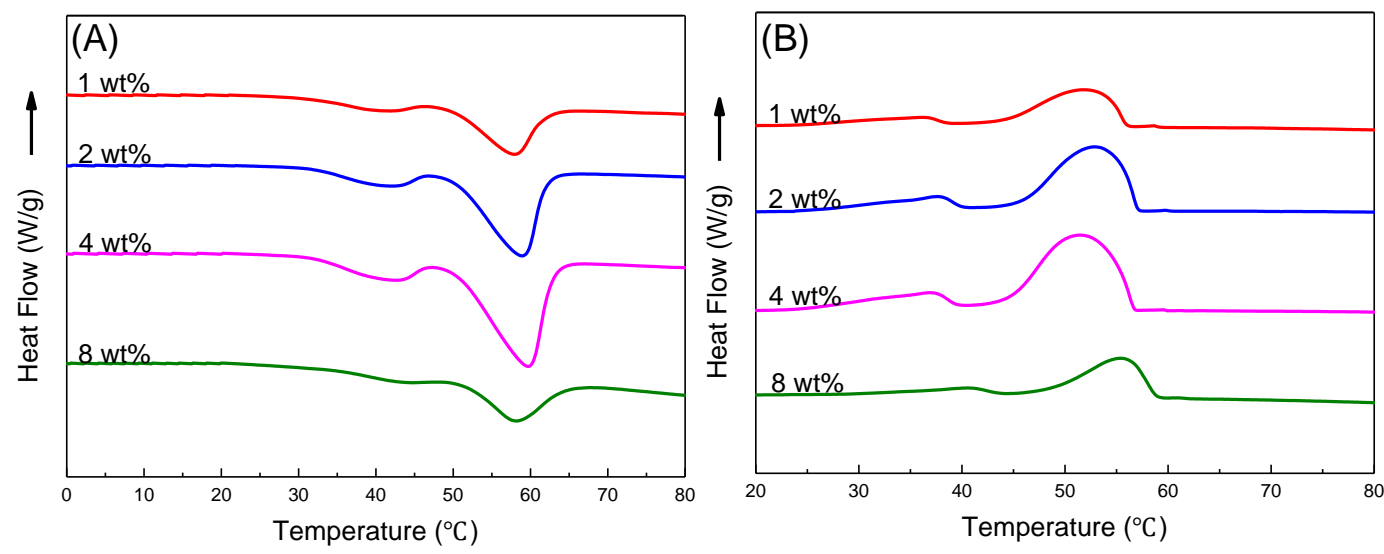

Figure 5. DSC curves of heating (A) and cooling (B) processes of microcapsules prepared with different amounts of sodium chloride. The heating and cooling rates are $10^{\circ} \mathrm{C} / \mathrm{min}$

Considering the melting enthalpy and morphology data, $4 \mathrm{wt} \%$ was the optimal amount of sodium chloride to obtain such microcrystalline paraffin/PUF microcapsules. The shape, separation degree, and surface smoothness the of microcapsules were remarkably improved as the content of sodium chloride increased. However, salt solution is also widely adopted for demulsion because hydration of inorganic salt can reduce free water and break the oil-in-water structure [42]. Therefore, the lower level of enthalpy seen in the microcapsules prepared with $8 \mathrm{wt} \%$ sodium chloride should be due to the hydration effect being of sodium chloride being, which reduced the encapsulation of paraffin. 


\subsection{Role of Sodium Chloride}

Two possible roles for inorganic salts during the preparation of the microcapsules have been put forward. One possible role is the synergistic effect. Salt and surfactant work together to decrease the critical micelles concentration, which helps increase the dispersibility and stability of emulsion. The other role is ionic strength. The addition of salt can increase the ionic strength of emulsion and help the colloidal form double layer as a stabilizer at a low concentration. The repulsive force between particles with the same kind of charge is strengthened, which helps the formation of larger droplets and easily keeps it not aggregate [43].

To verify the role of sodium chloride, paraffin emulsion with different amounts of sodium chloride were prepared and the emulsion size changing behaviors are shown in Figure 6. For each sample, emulsion size and the distribution width increased over time. Without the addition of sodium chloride, the average size of paraffin droplets was $0.49(0.21) \mu \mathrm{m}$ in the first hour, then it gradually increased and reached $1.72(0.54) \mu \mathrm{m}$ after stirring for $4 \mathrm{~h}$. The numbers in brackets indicate the distribution degrees of the droplet sizes. The situation was almost the same for emulsion with the addition of $1 \mathrm{wt} \%$ sodium chloride. However, the average size of emulsion visibly increased after adding $2 \mathrm{wt} \%$ sodium chloride. The emulsion size changed from $1.17(0.18) \mu \mathrm{m}$ to $2.15(0.70) \mu \mathrm{m}$ as the stirring time increased. Moreover, the size distribution became wider than the first two samples. With the addition of sodium chloride $4 \mathrm{wt} \%$, the emulsion size and size distribution further increased. The emulsion size increased from $2.79(0.92) \mu \mathrm{m}$ at 1 hour to $3.35(1.79) \mu \mathrm{m}$ at 4 hours. Therefore, we argue that sodium chloride possesses a strong effect on the morphology of paraffin emulsion, the enlargement of droplets, and the widening size of distribution, which should be attributed to the enhanced electric double layer strength.

A parameter of $\mathrm{D} / \mathrm{D}_{0}$ was performed and used to evaluate the stability of emulsion. $\mathrm{D}$ and $\mathrm{D}_{0}$ were the average sizes of emulsion droplets stirring at needed time and 1 hour, respectively. The larger $\mathrm{D} / \mathrm{D}_{0}$ value reflected the lower stability of emulsion. Interestingly, the $\mathrm{D} / \mathrm{D}_{0}$ value of paraffin emulsion without sodium chloride increased significantly with increasing stirring time and reached 3.51 at 4 hours. Therefore, we argue that the emulsion stability is rather poor and aggregation occurs remarkably, resulting in microcapsules with poor morphology. With the incorporation of sodium chloride, the increase level of $\mathrm{D} / \mathrm{D}_{0}$ declined and achieved a minimum state with $4 \mathrm{wt} \%$ sodium chloride. The $\mathrm{D} / \mathrm{D}_{0}$ value only increased to 1.2 after being stirred for 4 hours. Thus, we argue that sodium chloride improves the stability of paraffin emulsion, which benefits the obtainment of microcapsules with good morphology. When $8 \mathrm{wt} \%$ sodium chloride was introduced, the decrease of emulsion size and increase of $\mathrm{D} / \mathrm{D}_{0}$ value might be due to the hydration effect being too strong.

The electrostatic properties are important for emulsion stability as they can reduce or eliminate the influence of flocculation by increasing the repulsive forces between droplets [44,45]. The Zeta potentials of paraffin emulsion and prepolymer solution with different content of sodium chloride are shown in Figure 7. Despite a low value variation, the absolute Zeta potential of paraffin emulsion system increased and reached the peak at the sodium chloride content of $4 \mathrm{wt} \%$ with the increasing amount of sodium chloride. The repulsive forces effectively prevented the aggregation of droplets and improved their stability, leading to the rather steady size distribution of droplets (Figure 6D). Meanwhile, the Zeta potential of prepolymer reduced. Therefore, the ionic strength that increased with the addition of the appropriate level of sodium chloride can not only improve the electrical double layer structure of emulsions droplets but also weaken that of prepolymer. Furthermore, this shows that the post-polymerization can occur in more gentle and stable environments, thereby the encapsulation efficiency is improved. Therefore, we argue that $4 \mathrm{wt} \%$ sodium chloride is the optimal amount for preparing semi-crystalline paraffin/PUF microcapsules here. 

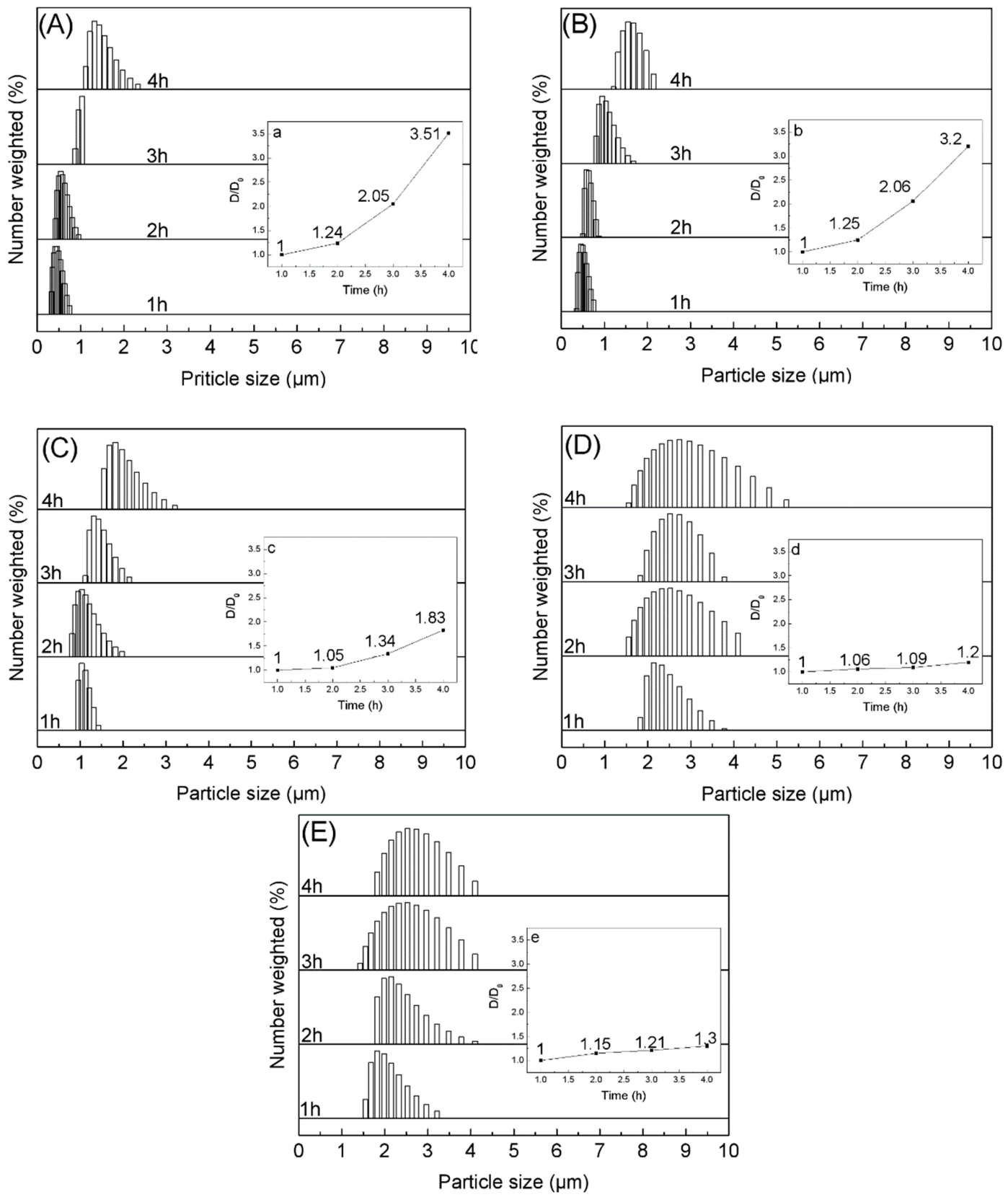

Figure 6. Dependence of emulsion size on the reaction stirring time for emulsifying system with different amounts of sodium chloride. 0 (A), $1 \mathrm{wt} \%$ (B), $2 \mathrm{wt} \%(\mathbf{C}), 4 \mathrm{wt} \%$ (D), and $8 \mathrm{wt} \%$ (E).

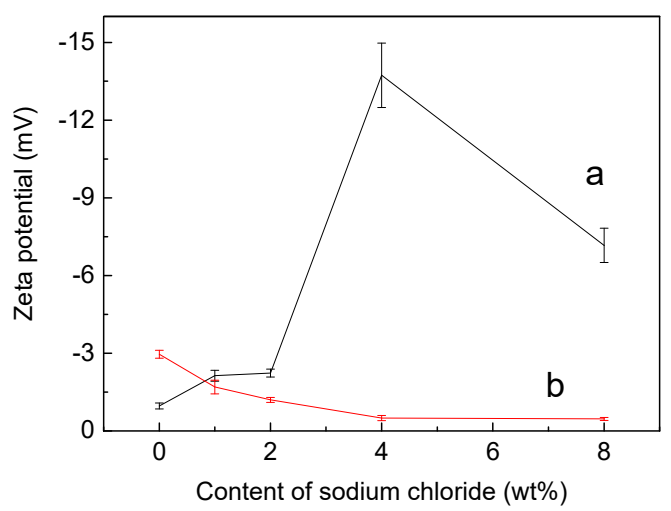

Figure 7. The Zeta potential of paraffin emulsion (a) and prepolymer solution (b). 


\subsection{Improve the Microcapsule Performance by Crosslinker}

With the optimum amount of sodium chloride, microcapsules with latent heat of $67.5 \mathrm{~J} / \mathrm{g}$ and good morphology were obtained. But it is still essential to improve thermal enthalpy. Therefore, a crosslinker was further applied to the optimum system above to improve capsulation efficiency, which also helped shorten the curing time. The crosslinker chosen was composed of resorcinol and ammonium chloride with a mass ratio of 1:1. Figure 8 shows the SEM images of microcapsules prepared with different amounts of crosslinker. The microcapsule spheres dispersed better when $0.25 \mathrm{wt} \%$ crosslinker was incorporated. Compared with Figure 4D, the smoothness of the surface of the microcapsules slightly decreased while the diameter hardly changed. With the addition of $0.35 \mathrm{wt} \%$ crosslinker, the microcapsules started to connect to each other and the morphology became worse. When the crosslinker content reached $0.5 \mathrm{wt} \%$, complete spheres begin to disappear, and independent spheres could not be observed. Only large size paraffin/PUF particles with irregular shapes and rather rough surfaces were obtained when $1 \mathrm{wt} \%$ crosslinker was introduced (Figure 8D).

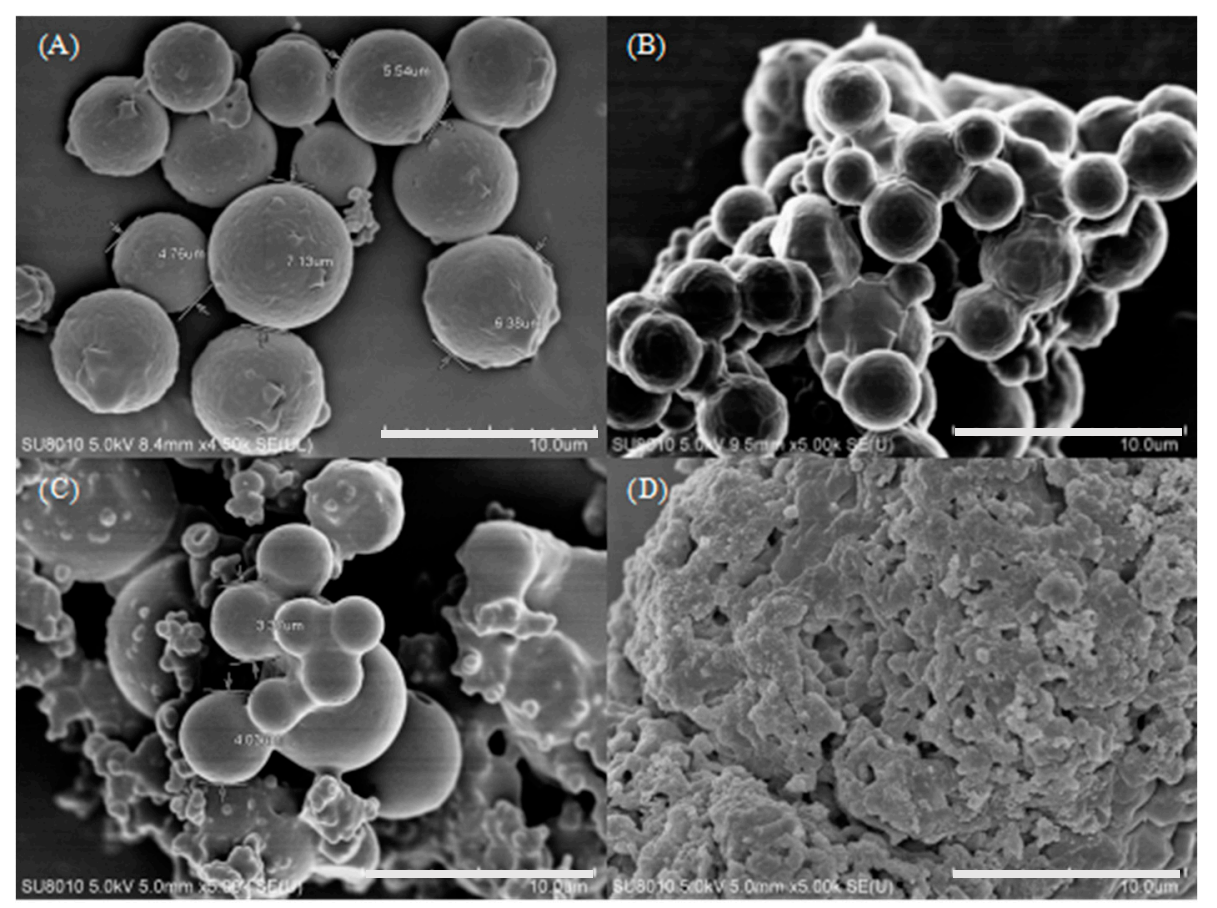

Figure 8. SEM images of microcapsules prepared with different amount of crosslinker. $0.25 \mathrm{wt} \%$ (A), $0.35 \mathrm{wt} \%$ (B), $0.5 \mathrm{wt} \%(\mathbf{C}), 1 \mathrm{wt} \%$ (D). The scale bars are all $10 \mu \mathrm{m}$.

The DSC curves of samples are presented in Figure 9. The melting enthalpy increased to $110.0 \mathrm{~J} / \mathrm{g}$ with the addition of $0.25 \mathrm{wt} \%$ crosslinker, which was an improvement of $63 \%$ when compared to microcapsules prepared without crosslinker. The enthalpy value further rose with more crosslinker and achieved $137.0 \mathrm{~J} / \mathrm{g}$ at crosslinker addition amount of $1 \mathrm{wt} \%$, which is a rather high value when compared to the literature $[25,26]$.

The two components, ammonium chloride and resorcinol, in as-used crosslinker played different roles in the polymerization process of the shell material. The main effect of ammonium chloride was to create an acidic condition in order to shorten the curing time. Ammonium chloride reacted with prepolymers as shown in formula (h) of Scheme 2 and the $\mathrm{pH}$ value was reduced. Figure 10 directly shows the measured $\mathrm{pH}$ value change of reaction system with different additions of crosslinker. The $\mathrm{pH}$ value gradually declined as the amount of crosslinker increased, the decline rate leveled off and reached 2.7. Normally, reduction of $\mathrm{pH}$ value can accelerate the curing process, which benefits the encapsulation of paraffin. However, excessive incorporation of crosslinker significantly reduces the $\mathrm{pH}$ value and leads to high reaction rate which goes against the formation of separated and spherical 
microcapsules. As shown in formula (i), resorcinol can directly participate in the polymerization process and increase connection between branches, which improves the crosslinking degree and capsulation efficiency. As one of the monomers, a small amount of resorcinol can generate positive effect but excess usage will affect the performance of PUF [46]. Thus, the crosslinker should be added after the prepolymers fully deposit on the surface of droplets, and the balanced addition amount of crosslinker for semi-crystalline paraffin/PUF microcapsules with well morphologies and high latent heat is $0.25 \mathrm{wt} \%$ here.
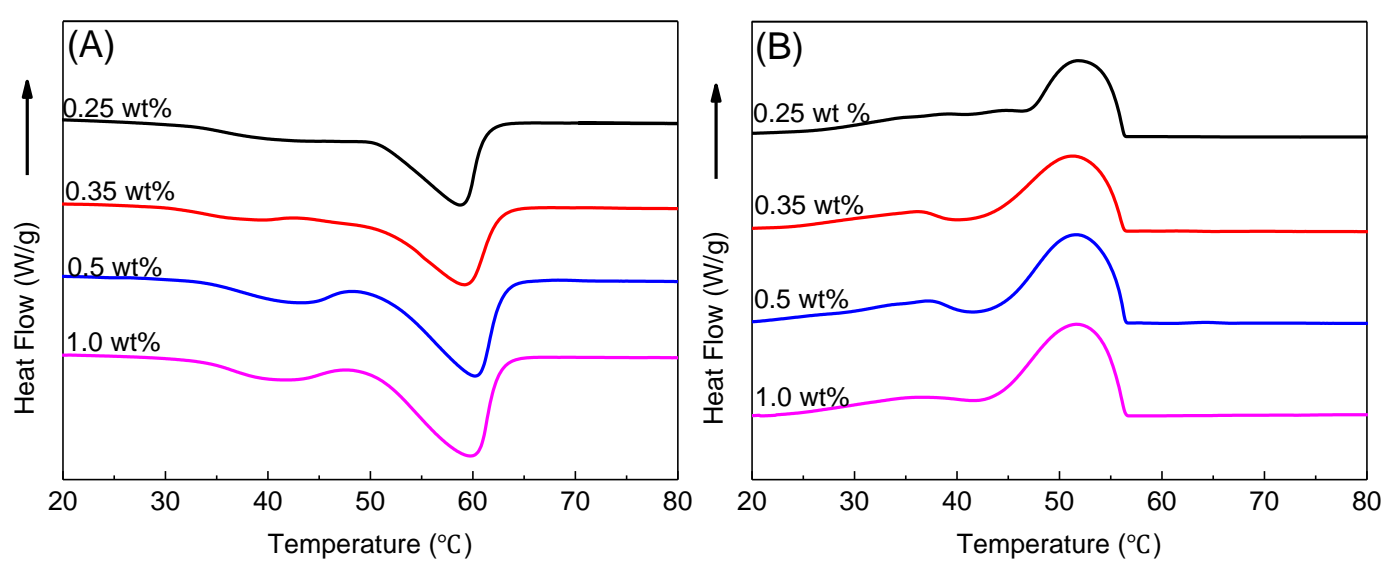

Figure 9. DSC curves of heating (A) and cooling (B) process of microcapsules prepared with further addition of different amount of crosslinker.<smiles>CN(C)C(=O)N(C)CO</smiles>

Scheme 2. Main reactions for ammonium chloride and resorcinol.

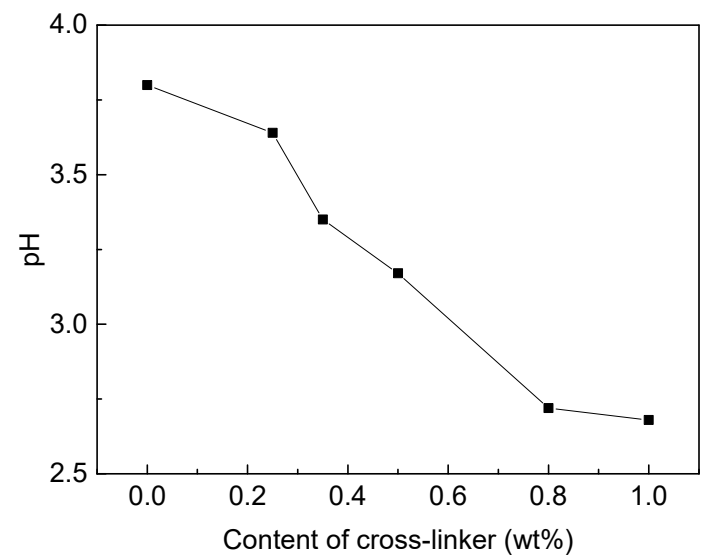

Figure 10. Dependence of reaction system $\mathrm{pH}$ value on the addition of crosslinker. 


\section{Conclusions}

In summary, semi-crystalline paraffin/PUF microcapsules with balanced morphology state and latent heat were successfully prepared by adding optimizing amounts of sodium chloride and crosslinker. The microcapsules showed a diameter of several microns and a melting enthalpy of $110 \mathrm{~J} / \mathrm{g}$, making it viable for TES application. The incorporation of sodium chloride strongly affected the morphology of paraffin emulsion by enlarging droplets, widening size distribution, and enhancing the stability, which should be attributed to the enhancement of electric double layer strength. In addition, sodium chloride has been shown to weaken the Zeta potential of prepolymer and provide more opportunity for prepolymer to deposit on the surface of emulsion droplets. The two components in crosslinker play different roles in synthesis process. Ammonium chloride has been shown to react with prepolymers and reduce the $\mathrm{pH}$ of system, which can accelerate the curing process. Resorcinol could directly participate in polymerization process and increase connection between branches.

Author Contributions: Data curation, K.Z.; Formal analysis, K.Z.; Funding acquisition, H.-M.Y.; Methodology, K.Z. and H.-M.Y.; Supervision, Q.Z. and H.-M.Y.; Writing—original draft, K.Z.; Writing—review \& editing, Q.Z. and H.-M.Y.

Funding: This research was funded by National Key Research and Development Plan (2016YFC0303708) and China University of Petroleum, Beijing.

Conflicts of Interest: The authors declare that they have no conflict of interest.

\section{References}

1. Zecca, A.; Chiari, L. Fossil-fuel constraints on global warming. Energy Policy 2010, 38, 1-3. [CrossRef]

2. Panwar, N.L.; Kaushik, S.C.; Kothari, S. Role of renewable energy sources in environmental protection: A review. Renew. Sustain. Energy Rev. 2011, 15, 1513-1524. [CrossRef]

3. Kannan, N.; Vakeesan, D. Solar energy for future world: A review. Renew. Sustain. Energy Rev. 2016, 62, 1092-1105. [CrossRef]

4. Agyenim, F.; Hewitt, N.; Eames, P.; Smyth, M. A review of materials, heat tranfer and phase change problem formulation for latent heat thermal energy storage systems. Renew. Sustain. Energy Rev. 2010, 14, 615-628. [CrossRef]

5. Dheep, G.R.; Sreekumar, A. Influence of nanomaterials on properties of latent heat solar thermal energy storage materials-A review. Energy Convers. Manag. 2014, 83, 133-148. [CrossRef]

6. Li, G. Energy and exergy performance assessments for latent heat thermal energy storage systems. Renew. Sustain. Energy Rev. 2015, 51, 926-954. [CrossRef]

7. de Gracia, A.; Cabeza, L.F. Phase change materials and thermal energy storage for buildings. Energy Build. 2015, 103, 414-419. [CrossRef]

8. Ascione, A.; Bianco, N.; de Masi, R.F.; de Rossi, F.; Vanoli, G.P. Energy refurbishment of existing builds through the use of phase change materials: Energy saving and indoor comfort in the cooling season. Appl. Energy 2014, 113, 990-1007. [CrossRef]

9. Gil, A.; Oró, E.; Miró, L.; Peiró, G.; Ruiz, A.; Salmerón, J.M.; Cabeza, L.F. Experimental analysis of hydroquinone used as phase change material to be applied in solar cooling refrigeration. Int. J. Refrig. 2014, 39, 95-103. [CrossRef]

10. Liu, M.; Saman, W.; Bruno, F. Development of a novel refrigeration system for refrigerated trucks incorporating phase change material. Appl. Energy 2012, 92, 336-342. [CrossRef]

11. Hassabo, A.G.; Mohamed, A.L.; Wang, H.; Popescu, C.; Moller, M. Metal salts rented in silica microcapsules as inorganic phase change materials for textile uesage. Inorg. Chem. 2015, 10, 59-65.

12. Shon, J.; Kim, H.; Lee, K. Improved heat storage rate for an automobile coolant waste heat recovery system using phase-change material in a fin-tube heat exchanger. Appl. Energy 2014, 113, 680-689. [CrossRef]

13. Tu, Y.; Zhu, W.; Lu, T.; Deng, Y. A novel thermoelectric harvester based on high-performance phase change material for space application. Appl. Energy 2017, 206, 1194-1202. [CrossRef]

14. Lu, W.; Tassou, S.A. Characterization and experiment investigation of phase change materials for chilled food refrigerated cabinet applications. Appl. Energy 2013, 112, 1376-1382. [CrossRef] 
15. Sharif, M.K.A.; Al-Abidi, A.A.; Mat, S.; Sopian, K.; Ruslan, M.H.; Sulaiman, M.Y.; Rosli, M.A.M. Review of the application of phase change material for heating and domestic hot water systems. Renew. Sustain. Energy Rev. 2015, 42, 557-568. [CrossRef]

16. Veerakumar, C.; Sreekumar, A. Phase change material based cold thermal energy storage: Materials, techniques, and applications-A review. Int. J. Refrig. 2016, 67, 271-289. [CrossRef]

17. Fang, G.; Chen, Z.; Li, H. Synthesis and properties of microencapsulated paraffin composites with $\mathrm{SiO}_{2}$ shell as thermal energy storage materials. Chem. Eng. J. 2010, 163, 154-159. [CrossRef]

18. Belessiotis, G.V.; Papadokostaki, K.G.; Favvas, E.P.; Efthimiadou, E.K.; Karellas, S. Preparation and investigation of distinct and shape stable paraffin $/ \mathrm{SiO}_{2}$ composite $\mathrm{PCM}$ nanospheres. Energy Convers. Manag. 2018, 168, 382-394. [CrossRef]

19. Li, B.; Liu, T.; Hu, L.; Wang, Y.; Gao, L. Fabrication and Propertied of microencapsulated paraffin@SiO $\mathrm{S}_{2}$ Phase Change Composite for Thermal Energy Storage. ACS Sustain. Chem. Eng. 2013, 1, 374-380. [CrossRef]

20. Zhan, S.; Chen, S.; Chen, L.; Hou, W. Preparation and characterization of polyurea microencapsulated phase change material by interfacial polycondensation method. Powder Technol. 2016, 292, 217-222. [CrossRef]

21. Rocha, G.A.; Fávaro-Trindade, C.S.; Grosso, C.R.F. Microencapsulation of lycopene by spray drying: Charcterization, stablity and application of microcapsules. Food Bioprod. Process. 2012, 90, 37-42. [CrossRef]

22. Gu, J.; Yang, X.; Li, C.; Kou, K. Synthesis of Cyanate Ester Microcapsules via Solvent Evaporation Technique and Its Application in Epoxy Resins as a Healing Agent. Ind. Eng. Chem. Res. 2016, 55, 10941-10946. [CrossRef]

23. Jacob, R.; Bruno, F. Review on shell materials used in the encapsulation of phase change materials for high temperature thermal energy storage. Renew. Sustain. Energy Rev. 2015, 48, 79-87. [CrossRef]

24. Gaitzsch, J.; Huang, X.; Voit, B. Engineering Functional Polymer Capsules toward Smart Nanoreactors. Chem. Rev. 2016, 116, 1053-1093. [CrossRef] [PubMed]

25. Cuomo, F.; Ceglie, A.; De Leonadis, A.; Lopez, F. Polymer Capsules for Enzymatic Catalysis in Confined Environments. Catalysts 2019, 9, 1. [CrossRef]

26. Giro-Paloma, J.; Martinez, M.; Cabeza, L.F.; Inés Fernández, A. Types, methods, techniques, and applications for miroencapsulated phase change materials: A review. Renew. Sustain. Energy Rev. 2016, 53, 1059-1075. [CrossRef]

27. Sánchez-Silva, L.; Lopez, V.; Cuenca, N.; Valverde, J.L. Poly(urea-formaldehyde) microcapsules containing commercial paraffin: In situ polymerization study. Colloid Polym. Sci. 2018, 296, 1449-1457. [CrossRef]

28. Jin, Z.; Wang, Y.; Liu, J.; Yang, Z. Synthesis and properties of paraffin capsules as phase change materials. Polymer 2008, 49, 2903-2910. [CrossRef]

29. Xin, C.; Tian, Y.; Wang, Y.; Huang, X. Effect of curing temperature on the performance of microencapsulated low melting point paraffin using urea-formaldehyde resin as a shell. Text. Res. J. 2014, 84, 831-839. [CrossRef]

30. Yan, Y.; Liu, J.; Zhang, H. Effects of $\mathrm{NaCl}$ and dispersants on preparation of microencapsulated phase change paraffin. New Chem. Mater. 2009, 37, 56-59.

31. Zhan, J.; Zou, D.; Li, L.; Ma, X.; Zhu, Y. Preparation and characterization of high temperature phase change paraffin microcapsules with urea-formaldehyde resin shell materials. Acta Mater. Compos. Sin. 2017, 34, 284-290.

32. Fang, G.; Li, H.; Yang, F.; Liu, X.; Wu, S. Preparation and characterization of nano-encapsulated n-tetradecane as phase change material for thermal energy storage. Chem. Eng. J. 2009, 153, 217-221. [CrossRef]

33. Fan, C.; Tang, J.; Zhou, X. Role of ammonium chloride in preparing poly(urea-formaldehyde) microcapsules using one-step method. J. Appl. Polym. Sci. 2013, 129, 2846-2856. [CrossRef]

34. Wang, H.; Hu, S.; Cai, S.; Yu, F. Preparation and properties of bisphenol A epoxy resin microcapsules coated with melamine-formaldehyde resin. Polym. Bull. 2014, 71, 2407-2419. [CrossRef]

35. Jiang, K.; Su, Y.; Xie, B.; Jiang, S.; Zhao, Y.; Wang, D. Effect of Geometrical Confinement on the Nucleation and Crystallization Behavior of n-Alkane Mixtures. J. Phys. Chem. B 2008, 112, 16485-16489. [CrossRef] [PubMed]

36. Sandomirski, K.; Walta, S.; Dubbert, J.; Allahyarov, E.; Schofield, A.B.; Löwen, H.; Richtering, W.; Egelhaaf, S.U. Heterogenrous crystallization of hard and soft spheres near flat and curved walls. Eur. Phys. J. Spec. Top. 2014, 223, 439-454. [CrossRef]

37. Steiger, M. Crystal growth in porous materials-II: Influence of crystal size on the crystallization pressure. J. Cryst. Growth 2005, 282, 470-481. [CrossRef] 
38. Fayyad, E.M.; Almaadeed, M.A.; Jones, A. Preparation and characterization of urea-formaldehyde microcapsules filled with paraffin oil. Polym. Bull. 2016, 73, 631-646. [CrossRef]

39. Qiao, Z.; Mao, J. Enhanced thermal properties with graphene oxide in the urea-formaldehyde microcapsules containing paraffin PCMs. J. Microencapsul. 2017, 34, 1-9. [CrossRef] [PubMed]

40. Huang, Z.; Yu, X.; Li, W.; Liu, S. Preparation of urea-formaldehyde paraffin microcapsules modified by carboxymethyl cellulose as a potential phase change material. J. For. Res. 2015, 26, 253-260. [CrossRef]

41. Su, W.; Darkwa, J.; Kokogiannakis, G. Review of solid-liquid phase change materials and their encapsulation technologies. Renew. Sustain. Energy Rev. 2015, 48, 373-391. [CrossRef]

42. Carpio, E.D.; Rodrígurz, S.; Rondóges, M.; Borges, B. Stability of water-Boscan crude oil emulsions: Effect of salts, alcohols and glycols. J. Pet. Sci. Eng. 2014, 122, 542-550. [CrossRef]

43. Kumar, S.; Mandal, A. Studies on interfacial behavior and wettability change phenomena by ionic and nonionic surfactants in presence of alkalis and salt for enhanced oil recovery. Appl. Surf. Sci. 2016, 372, 42-51. [CrossRef]

44. Li, C.; Mei, Z.; Liu, Q.; Wang, J.; Xu, J.; Sun, D. Formation and properties of paraffin wax submicron emulsions prepared by the emulsion inversion point method. Colloid Surf. A-Physicochem. Eng. Asp. 2010, 356, 1-3. [CrossRef]

45. Capek, I. Degradation of kinetically-stable o/w emulsions. Adv. Colloid Interface Sci. 2004, 107, 2-3. [CrossRef]

46. Cosco, S.; Ambrogi, V.; Musto, P.; Carfagna, C. Urea-Formaldehyde Microcapsules Containing an Epoxy Resin: Influence of Reaction Parameters on the Encapsulation Yield. Macromol. Symp. 2006, 234, 184-192. [CrossRef]

(C) 2019 by the authors. Licensee MDPI, Basel, Switzerland. This article is an open access article distributed under the terms and conditions of the Creative Commons Attribution (CC BY) license (http://creativecommons.org/licenses/by/4.0/). 